\title{
Malignancies after lung transplantation
}

\author{
Anne Olland ${ }^{1,2}$, Pierre-Emmanuel Falcoz ${ }^{1,2}$, Gilbert Massard $^{1,2}$ \\ ${ }^{1}$ Lung Transplantation Group, University Hospital Strasbourg, Strasbourg, France; ${ }^{2}$ INSERM (French institute for health and medical research) \\ 1260 Regenerative Nanomedecine, Translational Medicine Federation of Strasbourg, University of Strasbourg, Strasbourg, France \\ Contributions: (I) Conception and design: A Olland; (II) Administrative support: None; (III) Provision of study materials or patients: None; (IV) \\ Collection and assembly of data: A Olland; (V) Data analysis and interpretation: A Olland; (VI) Manuscript writing: All authors; (VII) Final approval \\ of manuscript: All authors. \\ Correspondence to: Dr. Anne Olland. Thoracic Surgery Department, 'Nouvel Hôpital Civil', University Hospital, 1 place de l'Hôpital, 67000 \\ Strasbourg, France. Email: anne.olland@chru-strasbourg.fr.
}

\begin{abstract}
Lung transplantation has become an efficient life-saving treatment for patients with end stage respiratory disease. The increasing good outcome following lung transplantation may be explained by growing experience of transplant teams and availability of potent immunosuppressive drugs. Nevertheless, the latter carries an inherent risk for malignancy besides other common side effects such as systemic hypertension, diabetes and renal dysfunction. Malignancies occur in a smaller proportion of patients but explain for a large proportion of deaths following transplantation. From the first year post-transplantation they will represent the third cause of death with an increasing incidence along post lung transplant survival. In this chapter, we will browse the different types of malignancies arising following lung transplantation. According to the different techniques for lung transplantation, specific types of bronchogenic carcinoma will be described in the explanted lung, in the native lung, and in the graft. Risk factors associated to immunosuppressive therapy, but also to occupational and environmental factors, especially smoking, will be discussed. Eventually, we will strive at integrating recommendations for the treatment of malignancies following lung transplantation.
\end{abstract}

Keywords: Lung transplantation; malignancies; immunosuppressive regimen; risk factors; bronchogenic carcinoma

Submitted Sep 23, 2017. Accepted for publication Apr 23, 2018.

doi: $10.21037 /$ jtd.2018.05.34

View this article at: http://dx.doi.org/10.21037/jtd.2018.05.34

\section{Introduction}

Relying on a clinical experience of more than 30 years, lung transplantation has become an efficient treatment for patients with end-stage respiratory disease. Overall median survival has reached 5.8 years regardless of the initial respiratory disease and the type of transplantation (1). When considering patients undergoing bilateral lung transplantation, conditional median survival for patients surviving the first year even has reached 9.8 years (1). These increasingly good outcomes may be explained by growing experience of transplant teams in long term management of patients, and availability of potent anti-infectious and immunosuppressive drugs.
While unconturnable to achieve long-term survival, immunosuppressive regimens also carry deleterious consequences for lung transplant recipients. Systemic hypertension arises in $80 \%$ of all patients surviving more than 5 years to lung transplantation; renal dysfunction, hyperlipidemia, and diabetes occur in $62 \%, 52 \%$, and $34 \%$ respectively. In comparison to the general population, cardiovascular comorbidities are more frequent in lung transplant recipients but represent the fourth cause of death only (1). On the opposite, malignancies occur in a lower proportion of patients (18\% of patients reaching 5 years survival, $28.7 \%$ of patients reaching 10 years survival) but are a stronger purveyor of mortality (1). Malignancies (lymphoma and solid organ malignancies) 
represent the third cause of death from the first-year posttransplant on (1). The two other main causes of death are graft related: chronic lung allograft dysfunction and infections. From the date of lung transplantation, the proportion of patients dying from malignancy is growing continuously with survival time, ranging from $3 \%$ of all patients during the first-year post-transplant to $14.5 \%$ after 5 years of survival (1). The opposite trends in comparison to the general population may be explained by specific risk factors related to lung transplantation.

\section{Immunosuppressive regimens}

Immunosuppressive therapy has been a key point in the development of lung transplantation: true survival following lung transplantation was only achieved in the early 80 s following the discovery of cyclosporine and increasing awareness for the need of a strong immunosuppression to maintain the lung graft and patients' survival (2).

So far, immunosuppressive regimen for lung transplantation is based on a three drugs regimen using calcineurin inhibitors, antimetabolites, and steroids. Two of the three main types of drugs used for lung transplantation carry a potential oncogenic effect of their own. Calcineurins inhibitors (CNI), such as cyclosporine and tacrolimus, inhibit DNA repair and apoptosis in damaged cells, and may support tumor progression by this pathway (3-5). CNI also inhibit cell adhesion, which acts in favor of cell migration or metastatic spread $(6,7)$. Azathioprine determines microsatellite DNA instability in myelodysplastic syndromes and may induce squamous cell carcinoma of the skin (8-10). Nevertheless, there is no evidence that steroids may enhance tumor progression. In specific situations of lymphoma, steroids may even reduce the tumor size and induce cell death.

On the opposite, newer drugs such as mTOR inhibitors have theoretical anti-tumor properties, although no clinical effect has ever been obviated in lung transplant recipients (11-13). While mTOR inhibitors are recommended to treat pleural Kaposi sarcoma in renal transplant recipients, attempts to modify lung transplant patients' regimen with introducing mTOR after solid organ cancer diagnosis did not affect the outcome or modify the natural history of disease (14). Despite some experimental evidence crediting antiproliferative properties to mTOR inhibitors, the relatively low caseload of lung transplant patients with solid organ cancer opposes to any clinical trial evaluating their potential contribution to cancer treatment.
Eventually, immunosuppression is a double-edged sword, which does not only favor development of cancer, but also adversely interferes with the efficacy of systemic antineoplastic treatment. By one aspect, immunosuppression will have deleterious consequences on the clinical state of the patient: renal dysfunction for example jeopardizes the possibilities for chemotherapy. Moreover, there is now experimental evidence showing that systemic treatment such as radiotherapy or chemotherapy are only efficient if they are associated to a competent immune system in the host organism. There is an effective span of time during which the given systemic therapy has its own direct effect on cancer. Once the administration of the therapy is over, the competent immune system maintains the result obtained on the tumor while relapse is observed rapidly in immunosuppressed organisms. The immunosuppressed state of lung transplant recipient may explain for the very short delays of relapse following systemic treatment for cancer (15-17).

\section{Post-transplant lymphoproliferative disease (PTLD)}

Regarding the risk for malignancies, immunosuppressive medications used for the regimen of lung transplant recipients will lead patients to a comparable state as HIV infection (18). Malignancies related to viral infections, such as Kaposi sarcomas or viral induced lymphomas, are favored. PTLD is caused by infection with oncogenic viruses, where the Epstein Barr virus (EBV) is the most prominent (19). The risk for PTLD increases with the cumulative level of immunosuppression. The first publication in 1984 showed the relation of PTLD with immunosuppression, but also the potential for regression of the disease when tapering the level of immunosuppression (20). Only a few non-EBV related cases have been reported. The prevalence of EBV infection is high: almost $95 \%$ of the general population have been infected, and most often in an asymptomatic fashion during childhood. Most patients will have experienced EBV infection at the time they qualify for lung transplantation. EBV has a tropism for naive B cells. In the immunocompetent host, EBV infection will summarize as a B cell infection turning B cells to proliferating B blasts, but the reaction of the competent immune system will turn them into B memory cells and let them quiet. On the opposite, immunosuppressive therapy will hamper the control of the immune system over infected B cells and allow them to replicate themselves. EBV DNA load will then be 
detectable in the peripheral blood (21). In the rare event of EBV negative patients at the time of lung transplantation, primary EBV infection after the start of immunosuppressive therapy is a serious threat, carrying a higher risk for PTLD $(22,23)$. In both scenarios, there is no validated EBV DNA load threshold to confirm PTLD on a pathognomonic basis (24). Confirmation of definitive diagnosis relies on the same strategy as for any lymphoproliferative disease. Treatment refers to usual chemotherapy protocols as per guidelines but needs to be associated with a tapering of immunosuppressive therapy (25). Comorbidities induced by long-lasting immunosuppression limit the application of chemotherapy.

\section{Skin malignancies}

Skin cancer following lung transplantation is another oncologic complication directly related to the strength of immunosuppression (26). Ducloux et al. demonstrated a correlation between a low titre of CD4 lymphocytes and the occurrence of skin carcinoma. Different organ transplants require different levels of immunosuppression: accordingly, incidence of skin carcinoma was lower in liver recipients, and higher in hearts transplants when compared to kidney recipients $(27,28)$. Similarly, patients submitted to three-drug regimens had a higher incidence of skin malignancies than recipients submitted to two-drugs regimens (27). According to a review of the UNOS registry, Magruder et al. obviated that skin cancer had been diagnosed in $16 \%$ out of 18,093 lung transplant recipients, for whom a three-drug regimen is mandatory (29). In comparison, only $7.2 \%$ of the same cohort developed any other type of de novo malignancy. The majority of skin lesions were squamous cell carcinomas. Co-carcinogenic factors do add to the risk represented by immunosuppressive drugs. These are viral infections with human papillomavirus infections on top, excessive sun exposure, and skin type (30).

There is still a controversy regarding the use of voriconazole; while considered effective to prevent aspergillosis, it acts as a potent photosensitizer $(31,32)$. Several studies obviated a higher incidence of skin carcinoma, especially of non-melanoma type, to the duration of voriconazole therapy. However, McLaughlin et al. identified confounding demographic and clinical factors which outweigh the relation between use of voriconazole and skin cancer to a non-significant level in lung and heart-lung recipients (33). Male gender, sun exposure, advanced age, history of COPD, and history of immune disorder were independent risk factors for nonmelanoma skin cancer after lung transplantation (33).

\section{Age at the time of lung transplantation}

In the general population, risk for cancer increases with age. Passing the threshold of 60 years of age, the risk for lung cancer doubles from $0.95 \%$ to $2.35 \%$ in men, and from $0.79 \%$ to $1.75 \%$ in women (34). The probability of developing cancer of any kind reaches $6 \%$ when patients cross the age of 50 years and reaches $14 \%$ when crossing the age of 60 (35). Gender related malignancies even have lower age limits: the probability for breast cancer in women or prostate cancer in men dramatically increases when crossing the threshold of 40 years of age (36). In the meantime, the proportion of lung transplant recipients older than 40 years has increased with time to more than $40 \%$. In addition, improved survival by era now leads younger recipients to cross the age landmarks. At least $30 \%$ of lung transplant recipients will live longer than 60 years of age (1).

\section{Occupational risk factors and lung disease}

Lung disease requiring lung transplantation and lung cancer share some common occupational and environmental risk factors.

\section{Smoking bistory}

Smoking is a major occupational risk factor. COPD and emphysema are the most common indications for lung transplantation in the ISHLT registry (1). In the recording of recipient's characteristics, COPD/emphysema is set apart from alpha-1 antitrypsin deficiency and represents $31 \%$ of all lung transplants (1). COPD/emphysema occurrence in patients is highly related to smoking history. Even in COPD/asthma overlap syndrome (ACOS), smoking history remains an important risk factor (37-39).

Recipient's and/or donor's smoking history is one of the main risk factors for primary lung carcinoma in lung transplant recipient. Primary lung carcinoma has been described in the native lung after single-lung transplantation, as well as in the allograft following singleor bilateral lung transplantation (40). In both cases, smoking history was identified as a risk factor. But smoking history is also a known risk factor for other malignancies such as bladder and urinary tract cancer, esophagus, pancreas, oral cavity, larynx, kidney, stomach, uterine cervix and acute 
myeloid leukemia (41). Smoking history is responsible for $30 \%$ of all cancer deaths in the United States (41). Approximately $60 \%$ to $70 \%$ of all candidates for lung transplantation have experienced smoking history $(42,43)$. Even in CF patients, some studies report up to $16 \%$ of former smokers (43).

\section{Occupational and environmental factors}

Some other occupational or environmental risk factors are related with the onset of lung fibrosis and possible lung carcinoma. Asbestosis and silicosis are both interstitial lung diseases that may lead to end-stage lung disease requiring transplantation and are risk factors for lung cancer at the same time. Even when not related to an environmental risk factor, lung fibrosis and lung disease related to systemic sclerosis may evolve to lung carcinoma based on a common ground of molecular pathogenic pathways (44). Fibrosis and lung interstitial disease represent the second most frequent indication for lung transplantation. All-together, COPD and fibrosis together represent more than $70 \%$ of all lung transplants each year and are a category of increased risk for lung cancer (1).

\section{Donor risk factors}

Risk for cancer brought in by the donor is due either to preexisting risk factors (especially risk factors for lung cancer), or to an evolutive neoplastic disease at the time of organ donation.

In the current context of shortage of donor lungs, it is well admitted to accept organs from donors with a documented exposure to risk factors such as moderate smoking or mineral particle inhalation, provided that donor work-up has excluded any abnormality. Lung cancer arising in the transplanted lung is so far an exceptional complication, concerning less than $2 \%$ of recipients (40). The appraisal of the real risk for cancer related to donor's smoking is so far controversial, but might become more obvious owing to steadily increasing survival after transplant. However, most published events of lung cancer after transplantation have been observed on lungs harvested from former smokers $(45,46)$.

Harvesting lungs from a donor with an active malignant tumour is an exceptional event. Two situations of brain death might be distinguished: either brain death occurs from an inner neural cause including brain tumors, or the brain death occurs from an outer neural cause. In the latter, donors with a known malignancy will be deemed unsuitable for solid organ donation. Indeed, most often, when a tumor is discovered during work-up or during the harvest procedure, the latter is stopped and all transplantations are cancelled. Only a few isolated cases of ongoing transplantation have been reported. The reasons to proceed with transplantation have been (1) high emergency status of the recipient and low estimated risk for metastases to the lung, (2) cancer which has not been identified at the time of lung harvesting, and (3) failure of recognizing cancer at frozen section analysis during harvest procedure (47). On theoretical grounds, circulating tumor cells at the time of harvest, or existing micrometastases in the donor organ might evolve towards gross metastases in the recipient subjected to immunosuppressive therapy (47). Considering solid organ donors with brain tumors, there is a proved case of a single donor with an active glioblastoma having transmitted tumors to three separate recipients (liver, kidney and lung) published in international literature (48). To prevent this dreadful complication, thorough clinical work-up before, and careful surgical examination of the whole donor during solid organ retrieval are mandatory (49). Some authors have proposed specific additional screening protocols for potential donors to rule out metastases in the harvested organs. Part of their recommendations may be easily applied such as millimetric body CT scan in all potential donors. But another part like performing systematic colonoscopy in donors older than 60 years may increase risks for donor loss (perforation) and costs (49). Though highly relevant for potential solid organ recipients, these recommendations still need to be validated on prospective clinical series of donor selection (49).

In the case of brain dead donors in which brain death is related to a brain tumor, the world health organization (WHO) classification based on the aggressiveness of central nervous system (CNS) tumors may help decide whether the donor should be proposed for solid organ harvesting or not (50). The risk of transmission is separated in 4 classes provided the pathological nature of the CNS tumor is known. According to the UNOS registry, low grade tumors have a low risk of transmission $(0.1 \%$ to $1 \%)$ whereas high grade tumor (III or IV in the WHO classification) have a high risk of transmission (over 10\%) (50-52). Despite low risk for grade I and II tumors ( $0.1 \%$ to $1 \%)$, the recipient should always be informed before performing solid organ transplantation (53). Despite the opportunity of accepting donors with low grade CNS tumors, only a $50 \%$ to $60 \%$ of patients dying from CNS tumors are accepted as solid 
organ donor representing $1 \%$ of all donors (54). This fact may come from donors with CNS tumors for which a recent pathologic diagnose is not available: either brain death is inaugural from a CNS tumor and pathologic diagnose was never made, or the donor has a medical story of CNS tumor with ancient pathologic examination but no details are available on the recent history of the tumor and the possibility of evolving towards a higher-grade tumor (53). In the setting of organ shortage, Beigee $e t$ al. propose to search for pathology results on the CNS tumors at the time of solid organ harvesting starting with a brain autopsy (53). Brain autopsy in the brain-dead heart beating donor is performed at first to send part or whole of the tumor to pathologic examination while the solid organ harvesting is carried out. In their publication, Beigee $e t$ al. report a 45 to 50 minutes process for pathologic diagnose on the CNS tumor. The short effective span of time enables rapid decision making on whether the transplantation process should be carried out or whether the harvested organs should be discarded. In their publication, brain autopsy first enabled the authors to enlarge their donor pool with 21 solid organs that would otherwise have been discarded facing the uncertain diagnose of the reported CNS tumors (53). One step further, in their retrospective review, Kashyap et al. evidenced no survival difference between their liver transplant recipients would they be transplanted with an organ from a CNS brain dead donor or not, whatever the grade of the involved CNS tumor (48). Among 42 liver transplant recipients from CNS donors, only one experienced recurrence from the CNS tumor but the patient eventually died from another cause (48). Liver transplantation requires a lower immunosuppressive regimen in comparison to lung transplantation, but in the end, clinical evidence may plead for a more tolerant attitude when considering CNS tumor brain dead donors. Indeed, when considering the ability of CNS tumors for metastatic spread, lung metastases are a rare occurrence. Less than $2 \%$ of all patients with CNS tumors present with extraneural metastases in the whole medical history of the CNS tumor. Moreover, lung metastases occur in the setting of an end stage evolutive CNS tumor with obvious clinical and radiological signs $(54,55)$.

\section{Scenarios for lung cancer in lung transplant recipients}

There are at least 3 different clinical situations: (I) lung cancer discovered on the explanted lung, (II) lung cancer in the native lung after single-lung transplantation, and (III) lung cancer in the transplanted lung of either type (40).

Lung cancer discovered on the explanted lung may be completely unexpected, but some recipients are accepted on waiting list with an undetermined, stable and PET negative nodule. Discovery of lung cancer on the explanted lung naturally arises anxiety about the potential for recurrence after transplantation. On the opposite, one might also consider that explantation of the lung is a potentially curative treatment of early stage cancer. The prognosis is determined by lymph node stage. However, node staging implicates complete node dissection, which is obviously not performed during lung transplantation. As a consequence, prognosis is uncertain in this category of patients. Nevertheless, there is some evidence that prognosis of early stage lung cancer does not differ from nontransplanted patients. On the opposite, prognosis is poor in patients with lymph node involvement, despite adjuvant chemotherapy (40).

The second scenario is bronchogenic carcinoma occurring in the native lung following single-lung transplantation. Single-lung transplantation is indicated for patients without chronic suppurative lung disease, and preferentially without pulmonary hypertension. In other words, most of the patients undergoing single-lung transplantation suffer from either COPD patients or fibrosis. Single-lung transplantation leaves behind a sick lung that has been exposed to risk factors for cancer, and smoking in particular; in addition, risk for lung cancer increases in case of idiopathic fibrosis, regardless of smoking history. Post-transplant immunosuppressive therapy adds to the risk. Bronchogenic carcinoma of the native lung is observed in 5-10\% of single-lung transplant recipients: as such, the risk for bronchogenic carcinoma is a 6-fold higher in comparison to patients undergoing bilateral lung transplantation $(40,56)$

Nevertheless, even bilateral lung transplantation does not wave away the risk for lung cancer in the graft. Bronchogenic carcinoma is observed in $1-2 \%$ of double lung transplant recipients and may arise from the graft as from the recipient himself. Spencer $e t a l$. demonstrated with repeated bronchial epithelium biopsies following bronchial suture that cells issued from the recipient bronchial epithelium are migrating towards the donor bronchi to form a mosaic with donor epithelial cells in up to $20 \%$ of the epithelial surface of the graft (57). These recipient cells may carry risk owing to previous smoking history of the recipient. The other origin for bronchogenic carcinoma 
comes from donor cells; there is so far no evidence defining a set-off in the quantification of smoking history. In some rare cases, the donor or recipient origin of cancer cells has been documented with in situ fluorescence hybridization assays, showing an almost even distribution.

Treatment of lung cancer after transplantation follows the guidelines applied to any patient with lung cancer. For early stage disease, surgery with curative intent should be considered. However, immunosuppressive therapy introduces a double limitation. As previously discussed, immunosuppressive drugs limit the efficiency of adjuvant therapies (15-17). Further, immunosuppressive drugs may lead to specific comorbidities, which may oppose to surgery and/or chemotherapy. Eventually, as the occurrence of cancer following lung transplantation also increases with duration of survival, the patient might suffer from chronic lung allograft dysfunction at the time of cancer diagnosis and be unfit for surgery on functional grounds.

\section{Final recommendations}

There aren't any specific recommendations available for malignancies following lung transplantation.

Considering the direct toxicity of immunosuppressive regimen, but also to the potent oncologic consequences, the equilibrium between benefits and adverse effects is critical. According to the current state of art, lung transplant recipients require a relatively harsh immunosuppression relying on a three-drug regimen (antimetabolite, steroids, and $\mathrm{CNI}$ ). But in some practices, the regimen may be reduced to two drugs by suppressing the antimetabolite. Besides, use of inhibitors of mTOR during the treatment of malignancies is still matter of debate. The question whether mTOR inhibitors should be added to the immunosuppressive regimen, or if they might substitute to one of the drugs, still needs to be answered with a dedicated trial.

Similarly, there are no recommendations whether the patient should undergo screening programs before lung transplantation or at the time when immunosuppression is started (58). Several authors describing lung cancer in the explanted lung made a retrospective review of chest CT scans of the patients just before lung transplantation. Only a few cases could have been diagnosed or at least suspected before lung transplantation. In most of the cases, there were unspecific findings suitable with infectious disease or lung consolidation, especially in patients with fibrosis $(59,60)$.

Once cancer has been diagnosed on the explanted lung, some author completed the staging with a complementary lymph node dissection performed through mediastinoscopy or even thoracoscopy. Adjuvant treatment was given following the usual guidelines for lung cancer.

If cancer has declared after lung transplantation, curative resection is the optimal choice, provided that respiratory function allows proceeding, and that comorbidity index is low. Obviously, prognosis is best for early stage.

Prevention of skin cancer relies on patients' education avoiding direct sun exposure, protecting the skin with sunscreens and appropriate clothing. A dermatologist should perform skin surveillance regularly for early detection and excision of premalignant lesions (30). Treatment should follow the usual guidelines for skin carcinoma. In case of melanoma, immunotherapy and interferon therapy may be applied according to the compatibility with immunosuppressive drugs, within the framework of a multidisciplinary team.

PTLD treatment is achieved by tapering the level of immunosuppression, associated to classic chemotherapy schemes. Some authors add mTOR inhibitors to reduce other associated drugs. In the few patients without EBV infection before transplantation, anti-viral therapy should be discussed to prevent primo-infection as it carries an even higher risk for PTLD.

For any other type of cancer, comprehensively speaking, medical work up and treatment should be performed according to the valid actual guidelines with respect to the suspected type of cancer.

\section{Acknowledgements}

None.

\section{Footnote}

Conflicts of Interest: The authors have no conflicts of interest to declare.

\section{References}

1. Yusen RD, Edwards LB, Kucheryavaya AY, et al. The Registry of the International Society for Heart and Lung Transplantation: Thirty-second Official Adult Lung and Heart-Lung Transplantation Report--2015; Focus Theme: Early Graft Failure. J Heart Lung Transplant 2015;34:1264-77.

2. Reitz BA, Wallwork JL, Hunt SA, et al. Heart- 
lung transplantation: successful therapy for patients with pulmonary vascular disease. $\mathrm{N}$ Engl J Med 1982;306:557-64.

3. Ahlers C, Kreideweiss S, Nordheim A, et al. Cyclosporin A inhibits Ca2pmediated upregulation of the DNA repair enzyme DNA polymerase beta in human peripheral blood mononuclear cells. Eur J Biochem 1999;264:952-9.

4. Pritchard DE, Singh J, Carlisle DL, et al. Cyclosporin A inhibits chromium(VI)-induced apoptosis and mitochondrial cytochrome c release and restores clonogenic survival in $\mathrm{CHO}$ cells. Carcinogenesis 2000;21:2027-33.

5. Hortelano S, López-Collazo E, Boscá L. Protective effect of cyclosporin A and FK506 from nitric oxide-dependent apoptosis in activated macrophages. Br J Pharmacol 1999;126:1139-46.

6. Hojo M, Morimoto T, Maluccio M, et al. Cyclosporine induces cancer progression by a cell-autonomous mechanism. Nature 1999;397:530-4.

7. Mohri T, Kameshita I, Suzuki S, et al. Rapid adhesion and spread of non-adherent colon cancer Colo201 cells induced by the protein kinase inhibitors, K252a and KT5720 and suppression of the adhesion by the immunosuppressants FK506 and cyclosporin A. Cell Struct Funct 1998;23:255-64.

8. Kasiske BL, Snyder JJ, Gilbertson DT, et al. Cancer after kidney transplantation in the United States. Am J Transplant 2004;4:905-13.

9. Swann PF, Waters TR, Moulton DC, et al. Role of postreplicative DNA mismatch repair in the cytotoxic action of thioguanine. Science 1996;273:1109-11.

10. Offman J, Opelz G, Doehler B, et al. Defective DNA mismatch repair in acute myeloid leukemia/ myelodysplastic syndrome after organ transplantation. Blood 2004;104:822-8.

11. Hussein MM, Mooij JM, Roujouleh HM. Regression of post-transplant Kaposi sarcoma after discontinuing cyclosporin and giving mycophenolate mofetil instead. Nephrol Dial Transplant 2000;15:1103-4.

12. Fernández A, Marcén R, Pascual J, et al. Conversion from calcineurin inhibitors to everolimus in kidney transplant recipients with malignant neoplasia. Transplant Proc 2006;38:2453-5.

13. Chiurchiu C, Carreño CA, Schiavelli R, et al. Results of the conversion to everolimus in renal transplant recipients with posttransplantation malignancies. Transplant Proc 2010;42:277-9.

14. Stallone G, Schena A, Infante B, et al. Sirolimus for
Kaposi's sarcoma in renal-transplant recipients. N Engl J Med 2005;352:1317-23.

15. Apetoh L, Tesniere A, Ghiringhelli F, et al. Molecular interactions between dying tumor cells and the innate immune system determine the efficacy of conventional anticancer therapies. Cancer Res 2008;68:4026-30.

16. Taniguchi K, Nishiura H, Yamamoto T. Requirement of the acquired immune system in successful cancer chemotherapy with cis-diamminedichloroplatinum (II) in a syngeneic mouse tumor transplantation model. J Immunother 2011;34:480-9.

17. Zitvogel L, Apetoh L, Ghiringhelli F, et al. The anticancer immune response: indispensable for therapeutic success? J Clin Invest 2008;118:1991-2001.

18. Serraino D, Piselli P, Busnach G, et al. Risk of cancer following immunosuppression in organ transplant recipients and in HIV-positive individuals in southern Europe. Eur J Cancer 2007;43:2117-23.

19. Verschuuren EAM. Post-Transplant lymphoproliferative syndrome and other malignancies. Eur Respir Mon 2009; 45:226-37.

20. Starzl TE, Nalesnik MA, Porter KA, et al. Reversibility of lymphomas and lymphoproliferative lesions developing under cyclosporin-steroid therapy. Lancet 1984;1:583-7.

21. Thorley-Lawson DA, Gross A. Persistence of the EpsteinBarr virus and the origins of associated lymphomas. N Engl J Med 2004;350:1328-37.

22. Walker RC, Paya CV, Marshall WF, et al. Pretransplantation seronegative Epstein-Barr virus status is the primary risk factor for posttransplantation lymphoproliferative disorder in adult heart, lung, and other solid organ transplantations. J Heart Lung Transplant 1995;14:214-21.

23. Haque T, Thomas JA, Falk KI, et al. Transmission of donor Epstein-Barr virus (EBV) in transplanted organs causes lymphoproliferative disease in EBV-seronegative recipients. J Gen Virol 1996;77:1169-72.

24. Stevens SJ, Verschuuren EA, Pronk I, et al. Frequent monitoring of Epstein-Barr virus DNA load in unfractionated whole blood is essential for early detection of posttransplant lymphoproliferative disease in high-risk patients. Blood 2001;97:1165-71.

25. Bakker NA, Verschuuren EA, Erasmus ME, et al. Epstein-Barr virus-DNA load monitoring late after lung transplantation: a surrogate marker of the degree of immunosuppression and a safe guide to reduce immunosuppression. Transplantation 2007;83:433-8.

26. Bouwes Bavinck JN, Hardie DR, Green A, et al. 
The risk of skin cancer in renal transplant recipients in Queensland, Australia. A follow-up study.

Transplantation 1996;61:715-21.

27. Jensen P, Hansen S, Møller B, et al. Skin cancer in kidney and heart transplant recipients and different longterm immunosuppressive therapy regimens. J Am Acad Dermatol 1999;40:177-86.

28. Frezza EE, Fung JJ, van Thiel DH. Non-lymphoid cancer after liver transplantation. Hepatogastroenterology 1997;44:1172-81.

29. Magruder JT, Crawford TC, Grimm JC, et al. Risk Factors for De Novo Malignancy Following Lung Transplantation. Am J Transplant 2017;17:227-38.

30. Euvrard S, Kanitakis J, Claudy A. Skin cancers after organ transplantation. N Engl J Med 2003;348:1681-91.

31. Feist A, Lee R, Osborne S, et al. Increased incidence of cutaneous squamous cell carcinoma in lung transplant recipients taking long-term voriconazole. J Heart Lung Transplant 2012;31:1177-81.

32. Zwald FO, Spratt M, Lemos BD, et al. Duration of voriconazole exposure: an independent risk factor for skin cancer after lung transplantation. Dermatol Surg 2012;38:1369-74.

33. McLaughlin JM, Equils O, Somerville KT, et al. Riskadjusted relationship between voriconazole utilization and non-melanoma skin cancer among lung and heart/lung transplant patients. Transpl Infect Dis 2013;15:329-43.

34. Jemal A, Siegel R, Xu J, et al. Cancer statistics, 2010. CA Cancer J Clin 2010;60:277-300. Erratum in: CA Cancer J Clin 2011;61:133-4.

35. Siegel RL, Miller KD, Jemal A. Cancer Statistics, 2017. CA Cancer J Clin 2017;67:7-30.

36. Siegel R, Naishadham D, Jemal A. Cancer statistics, 2013. CA Cancer J Clin 2013;63:11-30.

37. Postma DS, Rabe KF. The Asthma-COPD Overlap Syndrome. N Engl J Med 2015;373:1241-9.

38. Gelb AF, Nadel JA. Understanding the pathophysiology of the asthma-chronic obstructive pulmonary disease overlap syndrome. J Allergy Clin Immunol 2015;136:553-5.

39. Hines KL, Peebles RS Jr. Management of the AsthmaCOPD Overlap Syndrome (ACOS): a Review of the Evidence. Curr Allergy Asthma Rep 2017;17:15.

40. Olland AB, Falcoz PE, Santelmo N, et al. Primary lung cancer in lung transplant recipients. Ann Thorac Surg 2014;98:362-71.

41. Curran JN, Garrett-Mayer E, Carpenter MJ, et al. Cigarette smoking and the risk of dying from tobacco-related malignancies by race. Anticancer Res
2011;31:3891-5.

42. Hellemons ME, Agarwal PK, van der Bij W, et al. Former smoking is a risk factor for chronic kidney disease after lung transplantation. Am J Transplant 2011;11:2490-8.

43. Evon DM, Burker EJ, Sedway JA, et al. Tobacco and alcohol use in lung transplant candidates and recipients. Clin Transplant 2005;19:207-14.

44. Daniels CE, Jett JR. Does interstitial lung disease predispose to lung cancer? Curr Opin Pulm Med 2005;11:431-7.

45. Beyer EA, DeCamp MM, Smedira NG, et al. Primary adenocarcinoma in a donor lung: evaluation and surgical management. J Heart Lung Transplant 2003;22:1174-7.

46. Minai OA, Shah S, Mazzone P, et al. Bronchogenic carcinoma after lung transplantation: characteristics and outcomes. J Thorac Oncol 2008;3:1404-9.

47. Desai R, Collett D, Watson CJ, et al. Estimated risk of cancer transmission from organ donor to graft recipient in a national transplantation registry. Br J Surg 2014;101:768-74.

48. Kashyap R, Ryan C, Sharma R, et al. Liver grafts from donors with central nervous system tumors: a single-center perspective. Liver Transpl 2009;15:1204-8.

49. Hassanain M. Novel guidelines for organ donor cancer screening. Ann Transplant 2014;19:241-7.

50. Louis DN, Ohgaki H, Wiestler OD, et al. The 2007 WHO classification of tumours of the central nervous system. Acta Neuropathol 2007;114:97-109. Erratum in: Acta Neuropathol 2007;114:547.

51. Cancer Facts \& Figures 2018, American cancer society website. Available online: https://www.cancer.org/content/ dam/cancer-org/research/cancer-facts-and-statistics/ annual-cancer-facts-and-figures/2018/cancer-facts-andfigures-2018.pdf. Accessed April 2018.

52. Zülch KJ. Principles of the new World Health Organization (WHO) classification of brain tumors. Neuroradiology 1980;19:59-66.

53. Sadegh Beigee F, Shahryari S, Mojtabaee M, et al. Pathology Results at Autopsy in Brain-Dead Patients with Brain Tumors. Exp Clin Transplant 2017;15:113-5.

54. Lun M, Lok E, Gautam S, et al. The natural history of extracranial metastasis from glioblastoma multiforme. J Neurooncol 2011;105:261-73.

55. Beauchesne P. Extra-neural metastases of malignant gliomas: myth or reality? Cancers (Basel) 2011;3:461-77.

56. Dickson RP, Davis RD, Rea JB, et al. High frequency of bronchogenic carcinoma after single-lung transplantation. J Heart Lung Transplant 2006;25:1297-301. 
57. Spencer H, Rampling D, Aurora P, et al. Transbronchial biopsies provide longitudinal evidence for epithelial chimerism in children following sex mismatched lung transplantation. Thorax 2005;60:60-2.

58. Strollo DC, Dacic S, Ocak I, et al. Malignancies incidentally detected at lung transplantation: radiologic and pathologic features. AJR Am J Roentgenol 2013;201:108-16.

Cite this article as: Olland A, Falcoz PE, Massard G. Malignancies after lung transplantation. J Thorac Dis 2018;10(5):3132-3140. doi: 10.21037/jtd.2018.05.34
59. de Perrot M, Fischer S, Waddell TK, et al. Management of lung transplant recipients with bronchogenic carcinoma in the native lung. J Heart Lung Transplant 2003;22:87-9.

60. de Perrot M, Chernenko S, Waddell TK, et al. Role of lung transplantation in the treatment of bronchogenic carcinomas for patients with end-stage pulmonary disease. J Clin Oncol 2004;22:4351-6. 